\title{
Effects of dipeptidyl peptidase-4 inhibitor in insulin-resistant rats with myocardial infarction
}

\author{
Nattayaporn Apaijai1,2,3, Tharnwimol Inthachai', Suree Lekawanvijit1,4, \\ Siriporn C Chattipakorn 1,3,5 and Nipon Chattipakorn'1,2,3 \\ ${ }^{1}$ Cardiac Electrophysiology Research and Training Center, Faculty of Medicine, Chiang Mai University, \\ Chiang Mai, Thailand \\ ${ }^{2}$ Cardiac Electrophysiology Unit, Department of Physiology, Faculty of Medicine, Chiang Mai University, \\ Chiang Mai, Thailand \\ ${ }^{3}$ Center of Excellence in Cardiac Electrophysiology Research, Chiang Mai University, Chiang Mai, Thailand \\ ${ }^{4}$ Department of Pathology, Faculty of Medicine, Chiang Mai University, Chiang Mai, Thailand \\ ${ }^{5}$ Department of Oral Biology and Diagnostic Sciences, Faculty of Dentistry, Chiang Mai University, \\ Chiang Mai, Thailand
}

Correspondence should be addressed to $\mathrm{N}$ Chattipakorn

Email

nchattip@gmail.com

\begin{abstract}
Adverse cardiac remodeling after myocardial infarction (MI) leads to progressive heart failure. Obese-insulin resistance increases risks of $\mathrm{Ml}$ and heart failure. Although dipeptidyl peptidase-4 (DPP4) inhibitor is known to exert cardioprotection, its effects on adverse remodeling after $\mathrm{MI}$ in obese-insulin-resistant rats are unclear. We hypothesized that DPP4 inhibitor reduces adverse left ventricular (LV) remodeling and LV dysfunction in obese-insulin-resistant rats with MI. Rats were fed either normal diet (ND) or highfat diet (HFD) for 12 weeks to induce obese-insulin resistance, followed by left anterior descending coronary artery ligation to induce MI. Then, rats in each dietary group were divided into five subgroups to receive vehicle, enalapril ( $10 \mathrm{mg} / \mathrm{kg} /$ day), metformin (30 mg/kg/day), DPP4 inhibitor vildagliptin ( $3 \mathrm{mg} / \mathrm{kg} /$ day), or combined metformin and vildagliptin for 8 weeks. Heart rate variability (HRV), LV function, pathological and biochemical studies for LV remodeling, and cardiomyocyte apoptosis were determined. Obese-insulin-resistant rats had severe insulin resistance and LV dysfunction. HFD rats had a higher mortality rate than ND rats, and all treatments reduced the mortality rate in obese-insulin-resistant rats. Although all drugs improved insulin resistance, HRV, LV function as well as reduced cardiac hypertrophy and fibrosis, vildagliptin effectively reduced cardiomyocyte cross-sectional areas more than enalapril and was related to markedly decreased ERK $1 / 2$ phosphorylation. In ND rats with $\mathrm{MI}$, metformin neither improved LV ejection fraction nor reduced cardiac fibrosis. The infarct size and transforming growth factor- $\beta$ expression were not different among groups. In obese-insulin-resistant rats with chronic MI, DPP4 inhibitor vildagliptin exerts better cardioprotection than enalapril in attenuating adverse LV remodeling.
\end{abstract}

\section{Key Words}

- myocardial infarction

- vildagliptin

- metformin

- obese-insulin-resistant rats
Journal of Endocrinology (2016) 229, 245-258
() 2016 Society for Endocrinology Printed in Great Britain
Published by Bioscientifica Ltd 


\section{Introduction}

Heart failure is a major health problem that can be found in over 23 million people worldwide (Bui et al. 2011). A common cause of heart failure is coronary artery disease. Following myocardial ischemia due to coronary artery occlusion, left ventricular (LV) remodeling is known to occur. Postmyocardial infarction (MI) remodeling has been divided into early-phase (within $72 \mathrm{~h}$ post $\mathrm{MI}$ ) and late-phase LV remodeling (beyond $72 \mathrm{~h}$ ) (Sutton \& Sharpe 2000). During the late-phase LV remodeling, LV architecture is changed, including the distortion of LV shape, LV dilation, cardiomyocyte hypertrophy, scar formation, and increased wall stress, all of which could lead to LV dysfunction (Sutton \& Sharpe 2000).

It has been shown that long-term high-fat diet (HFD) consumption could induce obese-insulin resistance (Apaijai et al. 2012) and is related to an increased risk of MI (Oh et al. 2005), promotion of arrhythmic death, and worsening of LV dysfunction in a myocardial ischemia/ reperfusion (I/R) injury model (Apaijai et al. 2014, Liu et al. 2014). However, the roles of obese-insulin resistance in LV remodeling after MI are unclear. Furthermore, previous studies have shown that several oral antidiabetic drugs, including metformin and vildagliptin, exert cardioprotective effects against MI as well as heart failure in various animal models (Gundewar et al. 2009, Apaijai et al. 2014, Chinda et al. 2014).

Vildagliptin (a dipeptidyl peptidase- 4 inhibitor) is an oral antidiabetic drug that could reduce hyperglycemia in type 2 diabetes (Mari et al. 2005) and improve insulin sensitivity and cardiac sympathovagal balance in obeseinsulin-resistant rats (Apaijai et al. 2012, Pipatpiboon et al. 2013). In the heart, vildagliptin has been shown to exert beneficial effects in various models (Apaijai et al. 2013, Chinda et al. 2014, Miyoshi et al. 2014). It has been shown to preserve LV function and reduce the infarct size in cardiac I/R injury (Chinda et al. 2013, 2014, Apaijai et al. 2014). Moreover, vildagliptin reduced myocyte hypertrophy and perivascular and cardiac fibrosis in isoproterenol (Miyoshi et al. 2014), and transverse aortic constriction-induced heart failure (Takahashi et al. 2013). However, inconsistent findings exist as reported by Yin and colleagues who found that vildagliptin did not reverse LV remodeling in post-MI-induced heart failure rats (Yin et al. 2011). Moreover, the effects of vildagliptin, metformin, and combined vildagliptin and metformin on the heart with late-phase LV remodeling following MI in subjects with obese-insulin-resistant condition have not been investigated.
In this study, we investigated the effects of vildagliptin, metformin, and combined vildagliptin and metformin on cardiac sympathovagal balance, LV function, LV remodeling, including cardiomyocyte hypertrophy, cardiac fibrosis, and the molecular mechanisms responsible for these effects in rats with obese-insulin resistance. We hypothesized that combined vildagliptin and metformin exert superior cardioprotection against late-phase LV remodeling following MI in obeseinsulin-resistant rats compared with monotherapy.

\section{Materials and methods}

All experiments in this study were approved by the Institutional Animal Care and Use Committee of the Faculty of Medicine, Chiang Mai University, Chiang Mai, Thailand (permit no. 28/2557), in compliance with NIH and ARRIVE guidelines. Male Wistar rats weighing 180-200 g were obtained from the National Laboratory Animal Center, Salaya Campus, Mahidol University, Bangkok, Thailand. Rats were acclimatized for 7 days and housed in $12 \mathrm{~h}$ light: $12 \mathrm{~h}$ darkness cycle with controlled temperature $\left(25^{\circ} \mathrm{C}\right)$. Then, rats were divided into two dietary groups to receive either a normal diet (ND; a diet containing 19.8\% energy from fat) or an HFD (a diet containing 59.3\% energy from fat) for 12 weeks. At the 12th week, rats were subjected to permanent left anterior descending coronary artery (LAD) ligation to induce MI. Rats were allowed to acclimatize after surgery for 7 days when the inflammatory process of infarction was over and the scar formation was almost complete (Richer et al. 1999). After that, rats in each dietary group were subdivided into five subgroups $(n=6$ per group) to receive one of the following treatments: vehicle $(\mathrm{V} ; 0.9 \%$ normal saline solution in an equal volume), DPP4 inhibitor vildagliptin (Vil; $3 \mathrm{mg} / \mathrm{kg} / \mathrm{day}$, LAF 237, Novartis), metformin (M; $30 \mathrm{mg} / \mathrm{kg} /$ day, Glucophage, Merck Serono), combined vildagliptin and metformin (C), and enalapril (E; $10 \mathrm{mg} / \mathrm{kg} / \mathrm{day}$, served as positive control, Berlin, Bangkok, Thailand). Rats were treated with one of these drugs for 8 weeks via gavage feeding. These doses were selected based on our previous studies; our previous study showed that $3 \mathrm{mg} / \mathrm{kg} /$ day of vildagliptin, $30 \mathrm{mg} / \mathrm{kg} /$ day of metformin, and $10 \mathrm{mg} / \mathrm{kg} /$ day of enalapril exerted cardioprotection in various models, including obese-insulin-resistant rats (Apaijai et al. 2013), an I/R injury model (Apaijai et al. 2014), and normal rats with chronic MI (Inthachai et al. 2015).

Published by Bioscientifica Ltd. 


\section{Chronic MI model}

Rats were anesthetized using Zoletil $(0.3 \mathrm{~mL} / \mathrm{kg}$, Virbac Laboratories, Carros, France) and Xylazine $(0.075 \mathrm{~mL} / \mathrm{kg}$, Laboratorios Calier, S.A., Barcelona, Spain) via intramuscular injection. Surgery was performed under aseptic conditions, and thoracotomy was performed through the fourth intercostal space. The pericardium membrane was torn open, and the LAD was identified and ligated at $3 \mathrm{~mm}$ below its origin with 5-0 silk suture as previously described (Inthachai et al. 2015).

\section{Determination of metabolic parameters}

Body weight and food intake were recorded weekly. Blood samples were collected from the tail vein at baseline, week 12, and the end of treatment. Plasma glucose, total cholesterol, and triglyceride levels were determined using a commercial colorimetric assay kit (Erba Diagnostics Mannheim GmbH, Mannheim, Germany) (Apaijai et al. 2014). Plasma high-density lipoprotein (HDL) level was determined using a colorimetric assay kit (BioVision) (Apaijai et al. 2014). Plasma low-density lipoprotein (LDL) level was calculated using Friedewald equation (Apaijai et al. 2014). Plasma insulin levels were determined using a commercial ELISA kit (LINCO Research) (Pratchayasakul et al. 2011). The homeostatics model assessment (HOMA) index was used to assess insulin resistance, and the increased HOMA index indicates a higher degree of insulin resistance (Pratchayasakul et al. 2011). Plasma and cardiac 8-isoprostane levels were used as an oxidative stress marker and were determined using a commercial ELISA kit (OxiSelect, Cell Biolabs, Inc., San Diego, CA, USA) (Madian et al. 2011).

\section{Heart rate variability determination}

Heart rate variability (HRV) was used as an indicator of cardiac autonomic balance and was measured at baseline, week 4 , week 8 , week 12 , and at the end of treatment. A lead II electrocardiogram was recorded for $20 \mathrm{~min}$ in conscious rats using PowerLab (ADInstruments, Sydney, Australia) with Chart 5.0 program (Apaijai et al. 2013). Power spectra of R-R interval variability were obtained using fast Fourier transform algorithm. A high-frequency band (HF; $0.6-3 \mathrm{~Hz}$ ), a low-frequency band (LF; $0.2-0.6 \mathrm{~Hz}$ ), and a very-low-frequency band (below $0.2 \mathrm{~Hz}$ ) were analyzed using an analytical software program (Apaijai et al. 2013). Regarding the frequency domain analysis, the efferent vagal activity is a major contributor to the HF component, and the LF component represents both sympathetic and parasympathetic activities (Chattipakorn et al. 2007). The increased LF/HF ratio is considered as an index of cardiac sympathovagal imbalance (Chattipakorn et al. 2007).

\section{LV function determination}

Echocardiographic study Echocardiographic study was performed at baseline, week 4 , week 8 , week 12 , and the end of treatment. M-mode echocardiography was performed at the level of papillary muscles using Vivid-i (GE) under light anesthesia (2\% isoflurane with 98\% oxygen, Forane, Kent, UK). The percentage of fractional shortening $(\% \mathrm{FS})$ and the percentage of LV ejection fraction (\%LVEF) were determined (Apaijai et al. 2014).

Pressure-volume loop study This procedure was performed at the end of treatment. Rats were anesthetized using a combination of Zoletil $(50 \mathrm{mg} / \mathrm{kg})$ and Xylazine $(3 \mathrm{mg} / \mathrm{kg})$ via intramuscular injection. The right carotid artery was identified and the pressure-volume catheter (Scisense, London, Ontario, Canada) was inserted and advanced into the LV. End-systolic pressure, end-diastolic pressure (EDP), maximum and minimum $\mathrm{d} P / \mathrm{d} t$, and stroke volume to body weight ratio (SV/BW) were determined using an analytical software program (LabScribe, Dover, NH, USA) as previously described (Apaijai et al. 2013).

Blood pressure determination Blood pressure was measured at the end of treatment. A volume-pressure recording cuff and occlusion cuff were attached to the tail. Blood pressure measurement was performed using a CODA2 channel noninvasive blood pressure system (Kent Scientific Corporation, Torrington, CT, USA). Blood pressure values, including systolic and diastolic pressures, were achieved by taking an average of 20 consecutive measurements at a steady state (Lekawanvijit et al. 2012).

\section{Histological studies}

Infarct size and fibrosis determination Mid-LV cross sections were stained with picrosirius red and scanned (Aperio, Aperio Technologies Inc., San Diego, CA, USA) for infarct and fibrosis analysis. Infarct size was reported as the average of the proportions of endocardial and epicardial circumference by the infarct (Inthachai et al. 2015). Rats with nontransmural

Published by Bioscientifica Ltd. 
infarction were excluded from the study. Furthermore, picrosirius red-stained collagen matrix deposition from the whole LV myocardium was selected for its intensity, and the proportional area was calculated using a preset algorithm for picrosirius red stain intensity (Inthachai et al. 2015).

\section{Cardiomyocyte cross-sectional area deter-} mination Cardiomyocyte cross-sectional area was determined using hematoxylin and eosin (H\&E) staining. Fifty LV cardiomyocytes with equal-sized nuclei were randomly selected for analysis of cross-sectional area from prescanned images. Cell surface areas were calculated by measuring the circumferential length of the myocyte using Aperio ScanScope (Aperio, Aperio Technologies Inc., San Diego, CA, USA) (Inthachai et al. 2015).

Western blot analysis The myocardial tissues for western blot study were obtained from the fresh heart at the end of the treatment period following chronic MI. Myocardial protein extracts were prepared by the homogenization of nitrogen-frozen myocardial tissues in a $300 \mu \mathrm{L}$ extraction buffer containing $20 \mathrm{mM}$ Tris- $\mathrm{HCl}$ $(\mathrm{pH} 6.8), 1 \mathrm{mM}$ sodium orthovanadate, $5 \mathrm{mM}$ sodium fluoride, and a protease inhibitor. Total protein concentrations were determined using a Bio-Rad protein assay kit (Bio-Rad Laboratories). Sixty micrograms of total protein were mixed with the loading buffer $(5 \%$ betamercaptoethanol, $0.05 \%$ bromophenol blue, $75 \mathrm{mM}$ Tris- $\mathrm{HCl}(\mathrm{pH} 6.8), 2 \%$ SDS, and $10 \%$ glycerol) and loaded onto $10 \%$ SDS-acrylamide gels. Proteins were transferred to nitrocellulose membranes in a glycine/ methanol-transferred buffer in a wet/tank blotting system (Bio-Rad Laboratories). Membranes were blocked in 5\% skim milk in Tris-buffered saline and Tween buffer. Membranes were exposed to mouse polyclonal antirat BCL2, total connexin 43, p-connexin $43^{\text {ser368, total }}$ p38 mitogen-activated protein kinase (MAPK), p-p38

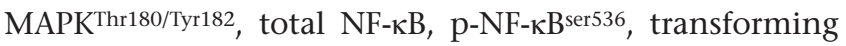
growth factor (TGF) $\beta$ (1:1000 dilution, Cell Signaling Technology), BAX, total ERK1/2, p-ERK1/2 Thr202/Tyr204, and $\beta$-actin (1:1000 dilution, Santa Cruz Biotechnology). Bound antibodies were detected using horseradish peroxidase conjugated with anti-rabbit IgG (1:2000 dilution, Cell Signaling Technology). The membranes were developed using the Clarity ECL Western Blotting Substrate (Bio-Rad) and densitometric analyses were carried out (Apaijai et al. 2014, Chinda et al. 2014).

\section{Statistical analysis}

Data were expressed as mean \pm s.E.M. One-way ANOVA followed by Tukey's post hoc test was used to determine the differences among groups. Time-course values of echocardiographic parameters and HRV were determined using two-way repeated measures ANOVA. Mortality rate was determined using $\chi^{2}$-test. A $P$-value of $<0.05$ was considered as statistically significance.

\section{Results}

Effects of enalapril, vildagliptin, metformin, and combined drugs on metabolic parameters

At baseline (week 0 before HFD consumption), there were no differences in body weight, food intake, plasma glucose, insulin, total cholesterol, LDL, HDL, triglyceride levels, and HOMA index between the ND- and HFDfed rats (Table 1). After 12-week HFD consumption, data showed that body weight, plasma insulin, total cholesterol, LDL levels, and HOMA index were increased in HFD-fed rats. However, there were no differences in food intake, plasma triglyceride, and HDL levels between the ND- and HFD-fed rats. Our data suggest that rats developed obese-insulin resistance after 12-week HFD consumption (Table 1).

Seven days after MI, rats received their assigned drugs for 8 weeks. At the end of treatment, we found that body weight was not changed in the ND and HFD groups, whereas LV/heart weight (HW) was increased in HFD-fed rats treated with vehicle (HFDV). All treatments reduced the LV/HW ratio in both MI rats; however, metformin did not reduce the LV/HW ratio in ND-fed rats (Table 2). Furthermore, we found that plasma glucose, insulin, and total cholesterol levels, and HOMA index were increased in HFDV compared with ND-fed rats treated with vehicle (NDV). Vildagliptin, metformin, and combined drugs reduced the levels of these parameters (Table 2).

\section{Effects of enalapril, vildagliptin, metformin, and combined drugs on oxidative stress levels}

We found that plasma and cardiac 8-isoprostane levels were higher in HFDV, compared with NDV. In ND-fed rats, treatment with enalapril, vildagliptin, and the combined drugs, but not metformin, reduced cardiac 8-isoprostane levels, whereas plasma 8-isoprostane level was not different among groups. In HFD-fed

Published by Bioscientifica Ltd 
Table 1 Effects of HFD consumption on metabolic parameters at baseline and 12 weeks after HFD consumption.

\begin{tabular}{|c|c|c|c|c|}
\hline \multirow[b]{2}{*}{ Metabolic parameters } & \multicolumn{2}{|c|}{ Baseline } & \multicolumn{2}{|c|}{ Week 12} \\
\hline & ND & HFD & ND & HFD \\
\hline Body weight (g) & $178 \pm 5$ & $181 \pm 3$ & $446 \pm 12$ & $533 \pm 10 *$ \\
\hline Food intake (g/day) & $15 \pm 2$ & $18 \pm 2$ & $19 \pm 2$ & $21 \pm 2$ \\
\hline Plasma glucose (mg/dL) & $128 \pm 4$ & $138 \pm 9$ & $122 \pm 10$ & $130 \pm 5$ \\
\hline Plasma insulin (ng/dL) & $2.4 \pm 0.4$ & $2.3 \pm 0.5$ & $2.6 \pm 0.3$ & $4.2 \pm 0.3^{*}$ \\
\hline HOMA index & $13 \pm 0.6$ & $14 \pm 0.1$ & $14 \pm 0.7$ & $24 \pm 0.2 *$ \\
\hline Plasma total cholesterol (mg/dL) & $54 \pm 6$ & $54 \pm 4$ & $53 \pm 6$ & $74 \pm 3^{*}$ \\
\hline Plasma triglyceride $(\mathrm{mg} / \mathrm{dL})$ & $123 \pm 5$ & $118 \pm 4$ & $119 \pm 1$ & $118 \pm 2$ \\
\hline Plasma HDL (mg/dL) & $20 \pm 5$ & $23 \pm 6$ & $24 \pm 4$ & $22 \pm 3$ \\
\hline Plasma LDL (mg/dL) & $9 \pm 6$ & $8 \pm 8$ & $7 \pm 2$ & $28 \pm 2^{*}$ \\
\hline
\end{tabular}

ND, normal diet-fed rats; HFD, high-fat diet-fed rats; HOMA, homeostatic model assessment; HDL, high-density lipoprotein; LDL, low-density lipoprotein. $* P<0.05$ vs ND.

rats, all treatments could reduce plasma and cardiac 8-isoprostane levels (Table 2).

\section{Effects of enalapril, vildagliptin, metformin, and} combined drug on HRV

At baseline, there was no difference in the LF/HF ratio between ND- and HFD-fed rats. The LF/HF ratio was increased after 8 weeks of HFD consumption, indicating the development of cardiac sympathovagal imbalance (Fig. 1A). After MI, HFDV rats had higher LF/HF ratio than NDV rats (Fig. 1B). In ND-fed rats, enalapril, vildagliptin, and combined drugs, but not metformin, reduced the $\mathrm{LF} / \mathrm{HF}$ ratio, whereas all treatments could reduce the LF/HF ratio in HFD-fed rats (Fig. 1B).

\section{Effects of enalapril, vildagliptin, metformin, and combined drug on LV function}

Echocardiographic parameters At baseline, there were no differences in the \%FS and \%LVEF between NDand HFD-fed rats. We found that \%FS and \%LVEF were decreased after 12-week HFD consumption (Fig. 2A and B). Furthermore, after treatment, HFDV demonstrated a worsened \%LVEF compared with NDV, whereas the $\%$ FS did not differ between HFDV and NDV rats (Fig. 2C and D). All treatments improved \%FS and \%LVEF in HFD-fed rats. However, metformin did not improve \%FS and \%LVEF in ND-fed rats compared with NDV rats (Fig. 2C and D).

Hemodynamic parameters At the end of treatment, only enalapril could reduce the systolic pressure in ND- and HFD-fed rats compared with NDV and HFDV rats (Table 3). Moreover, our data showed that HFDV rats had higher heart rate, higher EDP, and lower $\mathrm{d} P / \mathrm{d} t_{\min }$ compared with NDV rats. All treatments reduced EDP, and treatment with vildagliptin and combined drug increased $\mathrm{d} P / \mathrm{d} t_{\max }$ and $\mathrm{d} P / \mathrm{d} t_{\min }$ in HFD-fed rats. In ND-fed rats, treatment with vildagliptin and combined drug reduced EDP and increased $\mathrm{d} P / \mathrm{d} t_{\min }$, and enalapril could reduce only EDP. Metformin did not improve any hemodynamic parameters in NDfed rats (Table 3). Our data indicated that HFDV rats had worsened LV dysfunction than NDV rats, and all treatments improved LV function after MI in obese-insulin-resistant rats, whereas metformin did not improve any LV function parameters in ND-fed rats (Table 3 ).

Effects of enalapril, vildagliptin, metformin, and combined drug on mortality rate and $\mathrm{LV}$ remodeling For the perioperative mortality in the rats undergoing MI, the mortality rate was $27 \%$ in normal rats and $48 \%$ in obese-insulin-resistant rats during surgery. During 8 weeks of treatment period, we found that HFDV rats had a higher mortality rate after MI, and all treatments could reduce the mortality rate in HFD-fed rats (Fig. 3A). Our data also showed that connexin 43 (CX43) phosphorylation was lower in HFDV rats, and all treatment increased CX43 phosphorylation (Fig. 3B).

Infarct size, cardiac fibrosis, and cardiomyocyte cross-sectional area After MI, the infarct size did not differ between groups (Fig. 3C). We also determined an apoptotic (BAX) and antiapoptotic protein (BCL2) ratio, and found that BAX/BCL2 ratio was not different among groups (Fig. 3D).

In this study, we determined cardiomyocyte crosssectional areas from H\&E stained heart tissues, and

Published by Bioscientifica Ltd 
Table 2 Effects of enalapril, vildagliptin, metformin, and combination of vildagliptin and metformin on metabolic parameters in ND- and HFD-fed rats with chronic MI.

\begin{tabular}{|c|c|c|c|c|c|c|c|c|c|c|}
\hline Parameters & NDV & NDE & NDVil & NDM & NDC & HFDV & HFDE & HFDVil & HFDM & HFDC \\
\hline Body weight (g) & $545 \pm 3$ & $525 \pm 5$ & $475 \pm 8$ & $490 \pm 6$ & $520 \pm 4$ & $635 \pm 9 *$ & $620 \pm 5^{*}$ & $655 \pm 4 *$ & $610 \pm 8 *$ & $640 \pm 9 *$ \\
\hline LV/HW & $1.1 \pm 0.1$ & $0.7 \pm 0.2^{*}$ & $0.8 \pm 0.1 *$ & & $0.8 \pm 0.1 *$ & & & & & $0.8 \pm 0.1^{\dagger}$ \\
\hline $\begin{array}{l}\text { Visceral fat } \\
\text { weight (g) }\end{array}$ & $35 \pm 4$ & $41 \pm 5$ & $42 \pm 5$ & $44 \pm 7$ & $36 \pm 3$ & $68 \pm 4^{*}$ & & & & $68 \pm 7 *$ \\
\hline $\begin{array}{l}\text { Food intake } \\
\text { (g/day) }\end{array}$ & $17 \pm 1$ & $16 \pm 1$ & $17 \pm 1$ & $19 \pm 1$ & $15 \pm 1$ & $17 \pm 1$ & $14 \pm 1$ & $14 \pm 1$ & $15 \pm 1$ & $16 \pm 1$ \\
\hline $\begin{array}{l}\text { Plasma glucose } \\
\text { (mg/dL) }\end{array}$ & $130 \pm 4$ & $129 \pm 1$ & & $128 \pm 5$ & & $167 \pm 5^{*}$ & & $142 \pm 5^{\dagger}$ & $145 \pm 5^{\dagger}$ & $143 \pm 6^{+}$ \\
\hline $\begin{array}{l}\text { Plasma insulin } \\
\text { (ng/dL) }\end{array}$ & $3.0 \pm 0.2$ & $3.0 \pm 0.7$ & $2.8 \pm 0.4$ & $3.1 \pm 0.4$ & $3.1 \pm 0.4$ & $4.3 \pm 0.1 *$ & $3.9 \pm 0.2$ & & $2.7 \pm 0.2^{\dagger}$ & $3.3 \pm 0.3^{\dagger}$ \\
\hline HOMA index & $17 \pm 0.5$ & $17 \pm 0.2$ & & $18 \pm 0.7$ & $17 \pm 0.7$ & $32 \pm 0.9 *$ & $29 \pm 1$ & $22 \pm 0.7^{\dagger}$ & $20 \pm 0.6^{+}$ & $20 \pm 0.8^{\dagger}$ \\
\hline $\begin{array}{l}\text { Plasma total } \\
\text { cholesterol } \\
\text { (mg/dL) }\end{array}$ & & & & & & & & & & $113 \pm 2^{\dagger}$ \\
\hline $\begin{array}{l}\text { Plasma } \\
\text { triglyceride } \\
(\mathrm{mg} / \mathrm{dL})\end{array}$ & $120 \pm 5$ & $121 \pm 9$ & $135 \pm 6$ & $118 \pm 7$ & $133 \pm 10$ & $123 \pm 10$ & $124 \pm 10$ & $119 \pm 5$ & $122 \pm 6$ & $129 \pm 5$ \\
\hline $\begin{array}{l}\text { Plasma HDL } \\
\text { (mg/dL) }\end{array}$ & $24 \pm 2$ & $28 \pm 1$ & $27 \pm 1$ & $28 \pm 2$ & $28 \pm 2$ & $24 \pm 1$ & $25 \pm 2$ & $26 \pm 2$ & $25 \pm 3$ & $24 \pm 1$ \\
\hline $\begin{array}{l}\text { Plasma LDL } \\
\text { (mg/dL) }\end{array}$ & $12 \pm 4$ & $13 \pm 6$ & $14 \pm 2$ & $16 \pm 3$ & $16 \pm 3$ & $102 \pm 5^{*}$ & $65 \pm 3^{+}$ & $67 \pm 2^{+}$ & $64 \pm 3^{+}$ & $63 \pm 3^{+}$ \\
\hline $\begin{array}{l}\text { Plasma 8-isopros- } \\
\text { tane }(\mathrm{ng} / \mathrm{mL})\end{array}$ & $2.8 \pm 0.2$ & $2 \pm 0.2$ & $2.1 \pm 0.1$ & $2.7 \pm 0.2$ & $2.0 \pm 0.1$ & $3.4 \pm 0.3 *$ & $2.3 \pm 0.1^{\dagger}$ & $2.2 \pm 0.2^{\dagger}$ & $2.5 \pm 0.1^{\dagger}$ & $2.3 \pm 0.1$ \\
\hline $\begin{array}{l}\text { Cardiac 8-iso- } \\
\text { prostane } \\
(\mathrm{ng} / \mathrm{mg})\end{array}$ & $3.4 \pm 0.1$ & $2.9 \pm 0.1$ * & $2.7 \pm 0.1$ * & $3.4 \pm 0.3$ & $2.4 \pm 0.1$ * & $3.9 \pm 0.1$ * & $3.1 \pm 0.2^{\dagger}$ & $3.1 \pm 0.2^{+}$ & $3.1 \pm 0.1^{\dagger}$ & $2.9 \pm 0.1^{\dagger}$ \\
\hline
\end{tabular}

LV/HW, left-ventricular weight/heart weight; HOMA, homeostatic model assessment; HDL, high-density lipoprotein; LDL, low-density lipoprotein; NDV, normal diet rats treated with vehicle; NDE, normal diet rats treated with enalapril; NDVil, normal diet rats treated with vildagliptin; NDM, normal diet rats treated with metformin; NDC, normal diet rats treated with combined drugs; HFDV, high-fat-fed rats treated with vehicle; HFDE, high-fat-fed rats treated with enalapril; HFDVil, high-fat-fed rats treated with vildagliptin; HFDM, high-fat-fed rats treated with metformin; HFDC, high-fat-fed rats treated with combined drugs.

${ }^{*} P<0.05$ vs NDV, ${ }^{+} P<0.05$ vs HFDV.

cardiac fibrosis from picrosirius-stained heart tissues (Fig. 4A, B, C and D). Our results showed that cardiac fibrosis and cardiomyocyte cross-sectional areas were increased in HFDV rats, and all treatments reduced cardiomyocyte cross-sectional areas in HFD-fed rats (Fig. 4A, B, C and D). However, only metformin did not reduce cardiomyocyte cross-sectional areas in ND-fed rats. Interestingly, vildagliptin and combined drug treatment markedly reduced cardiomyocyte crosssectional areas compared with enalapril in both NDand HFD-fed rats (Fig. 4B). Furthermore, all treatments decreased cardiac fibrosis in HFD-fed rats, whereas metformin did not reduce cardiac fibrosis in ND-fed rats (Fig. 4A, B, C and D).

Our results showed that all treatments could reduce p38 phosphorylation in HFD-fed rats (Fig. 5A). In ND-fed rats, only metformin did not reduce p38 phosphorylation (Fig. 5A). ERK1/2 phosphorylation tended to increase in HFDV rats, and only vildagliptin reduced ERK1/2 phosphorylation in both ND- and HFD-fed rats (Fig. 5B).
Furthermore, we found that all treatments decreased NF-kB phosphorylation in HFD-fed rats but not in ND-fed rats (Fig. 5C). TGF- $\beta$ expression was not different among groups (Fig. 5D).

\section{Discussion}

The major findings of this study are as follows: vildagliptin, metformin, and combined metformin and vildagliptin effectively attenuated the insulin-resistant condition in obese-insulin-resistant rats. Furthermore, vildagliptin, metformin, combined drugs, and enalapril reduced plasma and cardiac oxidative stress, improved HRV, and attenuated LV dysfunction and LV remodeling in obeseinsulin-resistant rats. However, vildagliptin reduced cardiac hypertrophy better than other regimens in this model. In ND-fed rats, both vildagliptin and enalapril exerted cardioprotection against adverse LV remodeling. However, metformin exerted no benefit on late-phase LV remodeling after MI in ND-fed rats. 

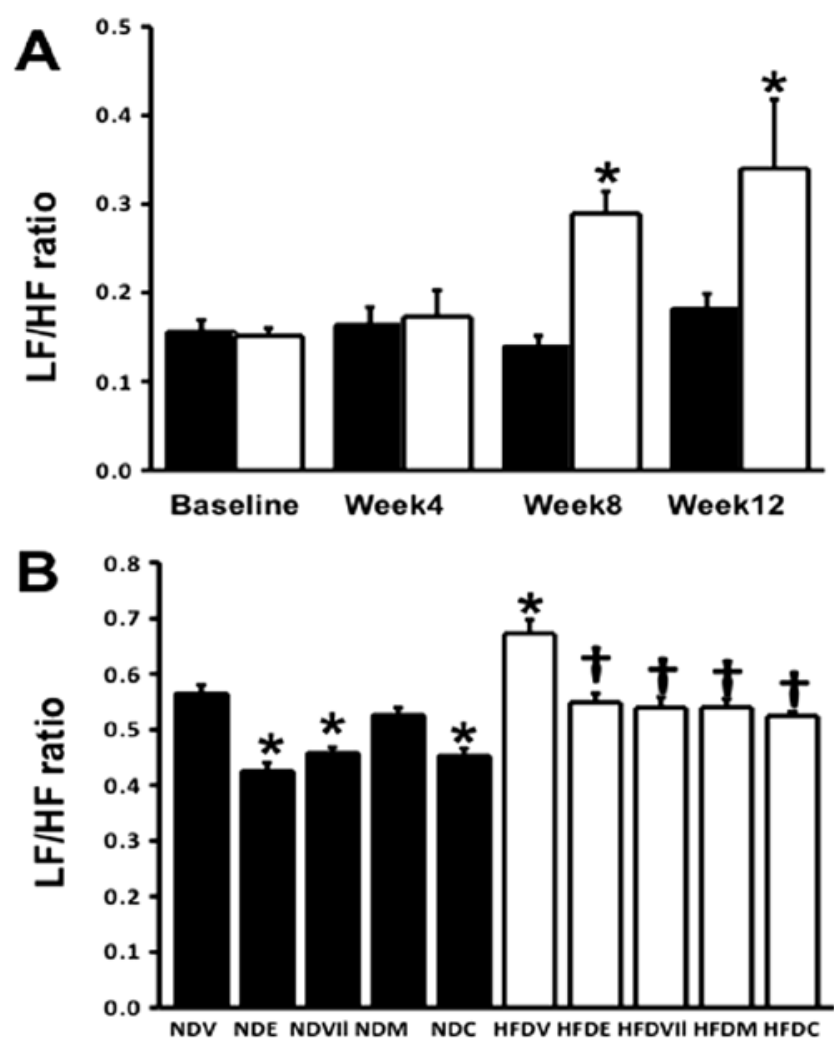

Figure 1

Effects of enalapril, vildagliptin, metformin, and combined drugs on HRV in normal and obese-insulin-resistant rats after chronic MI. The LF/HF ratio was increased after 8 weeks of HFD consumption. (A) $* P<0.05$ vs ND. Enalapril, vildagliptin, metformin, and combined vildagliptin and metformin improved HRV in obese-insulin-resistant rats. In ND rats, enalapril, vildagliptin, and combined vildagliptin and metformin but not metformin reduced the LF/HF ratio. (B) $* P<0.05$ vs NDV, ${ }^{+} P<0.05$ vs HFDV. ND, normal diet-fed rats; HFD, high-fat diet fed rats; LF, low-frequency; $H F$, high-frequency; NDV, normal diet rats treated with vehicle; NDE, normal diet rats treated with enalapril; NDVil, normal diet rats treated with vildagliptin; NDM, normal diet rats treated with metformin; NDC, normal diet rats treated with combined drugs; HFDV, high-fat-fed rats treated with vehicle; HFDE, high-fat-fed rats treated with enalapril; HFDVil, high-fat-fed rats treated with vildagliptin; HFDM, high-fat-fed rats treated with metformin; HFDC, high-fat-fed rats treated with combined drugs.

After chronic MI, obese-insulin-resistant rats exhibited severe insulin resistance by increased HOMA index and plasma glucose level. Our data demonstrated that vildagliptin, metformin, and combined drugs improved insulin resistance without hypoglycemia. This finding is consistent with previous studies, in which these drugs improved insulin resistance in patients with type 2 diabetes mellitus (T2DM) and in animal models of insulin resistance without MI (Bailey 2005, Apaijai et al. 2013, Pintana et al. 2013, Derosa et al. 2014). Furthermore, our data suggested that vildagliptin and metformin also effectively improved metabolic parameters in an

animal model of obese-insulin resistance with chronic MI (Fig. 6).

In this study, only enalapril, an angiotensin-converting enzyme (ACE) inhibitor reduced systolic blood pressure and associated with reduced LDL cholesterol levels in obeseinsulin-resistant rats. Clinical trials reported that enalapril reduced blood pressure by reduced angiotensin II level (Ocaranza et al. 2006) and LDL cholesterol level (Rachmani et al. 2000). Furthermore, the lower level of angiotensin II in addition to lower LDL cholesterol level could induce vasodilation (Kiliszek et al. 2007) and reduce systolic blood pressure. However, vildagliptin, metformin, and combined metformin and vildagliptin also reduced LDL cholesterol level without any changes in blood pressure. This finding consistent with previous reports in the heart failure model in which vildagliptin did not change blood pressure in rats with cardiac hypertrophy (Miyoshi et al. 2014), and metformin did not affect blood pressure and flow-mediated vasodilation in insulin-resistant patients with chronic HF (Wong et al. 2012). These data suggest that a reduction of LDL cholesterol level along with lower angiotensin II is necessary to reduce blood pressure in obese-insulinresistant rats with chronic MI.

HRV is an indicator of cardiac sympathovagal balance as well as a predictor of sudden cardiac death after MI (Zuanetti et al. 1996, Chattipakorn et al. 2007), and depressed HRV is commonly found in MI patients (Chattipakorn et al. 2007). In our study, a depressed HRV was worsening in obese-insulin-resistant rats with chronic MI than in ND rats. Vildagliptin, metformin, combined vildagliptin and metformin, and enalapril improved HRV in obese-insulin-resistant rats. Vildagliptin, combined drugs, and enalapril, but not metformin, also improved HRV in ND rats. This finding was correlated with the reduction of oxidative stress level. Our data were consistent with several studies which reported that an increased oxidative stress level has a positive correlation with a degree of cardiac sympathovagal imbalance in insulin-resistant patients and in animal models (Manzella et al. 2001, Pavithran et al. 2008, Apaijai et al. 2013). This could be the explanation why vildagliptin and enalapril, but not metformin, improved HRV in ND-fed rats with MI because metformin did not reduce oxidative stress in this group (Fig. 6).

LV remodeling, including LV hypertrophy and cardiac fibrosis, is a common phenomenon that occurs following MI (Sutton \& Sharpe 2000). In this study, vildagliptin, metformin, combined drugs, and enalapril reduced LV remodeling in obese-insulin-resistant rats. It is interesting that vildagliptin exerted better effect on LV remodeling because it could reduce cardiomyocyte hypertrophy

Published by Bioscientifica Ltd. 

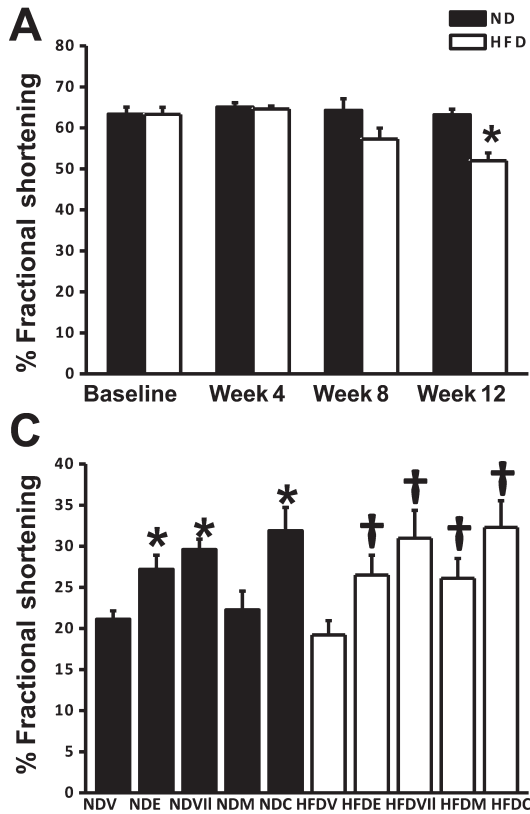

B

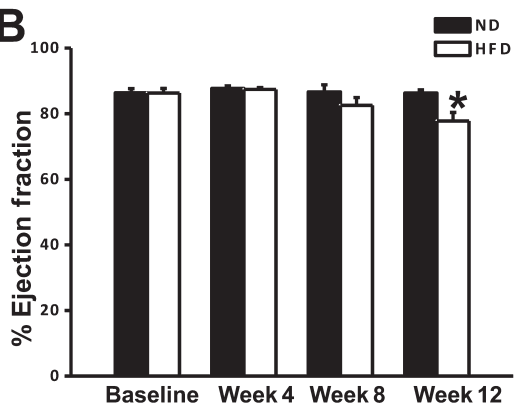

D

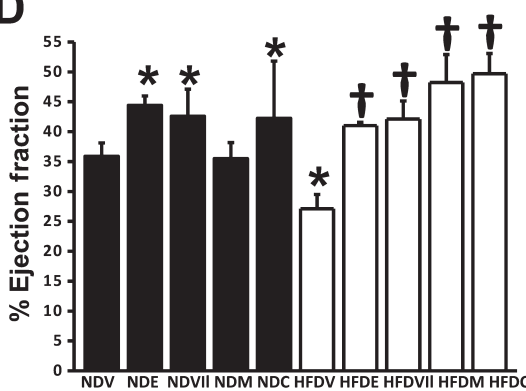

Figure 2

Effects of enalapril, vildagliptin, metformin, and combined drugs on \% FS and \%LVEF in normal and obese-insulin-resistant rats after chronic MI. \%FS and \%LVEF were decreased after 12 -week of HFD consumption ( $A$ and $B$ ), $* P<0.05$ vs ND. Enalapril, vildagliptin, metformin, and combined vildagliptin and metformin increased \%FS and \%LVEF in obese-insulin-resistant rats. In ND rats, enalapril, vildagliptin, and combined vildagliptin and metformin but not metformin increased $\% \mathrm{FS}$ and $\%$ LVEF (C and D), ${ }^{*} P<0.05$ vs NDV, ${ }^{+} P<0.05$ vs HFDV. ND, normal diet-fed rats; HFD, high-fat-diet fed rats; NDV, normal diet rats treated with vehicle; NDE, normal diet rats treated with enalapril; NDVil, normal diet rats treated with vildagliptin; NDM, normal diet rats treated with metformin; NDC, normal diet rats treated with combined drugs; HFDV, high-fatfed rats treated with vehicle; HFDE, high-fat-fed rats treated with enalapril; HFDVil, high-fatfed rats treated with vildagliptin; HFDM, high-fat-fed rats treated with metformin; HFDC high-fat-fed rats treated with combined drugs. greater than other regimens. Unlike our findings in obeseinsulin-resistant rats, a previous study in normal rats with MI reported otherwise. Yin and colleagues reported that vildagliptin reduced neither cardiomyocyte size nor LV dysfunction when given at 3 weeks after MI (Yin et al. 2011), whereas our data suggested that vildagliptin exerted cardioprotection when given 7 days after MI in both normal and obese-insulin-resistant rats. This disparity in results could be influenced by the timing of drug administration and the models used in those studies. In this study, the mechanism responsible for the reduction of cardiomyocyte hypertrophy by vildagliptin was related to decrease ERK and p38 MAPK phosphorylation. ERK and p38 MAPK are the members of MAPK, which are activated by oxidative stress, and both ERK and p38 MAPK also regulate cardiomyocyte hypertrophy during LV remodeling. Yeh and colleagues reported that ERK phosphorylation was increased in mice after 4-week post MI and related with cardiac hypertrophy (Yeh et al. 2010). Vildagliptin and combined vildagliptin and metformin reduced ERK phosphorylation, thus attenuating cardiomyocyte hypertrophy in our rat model of obeseinsulin-resistant and normal rats with chronic MI (Fig. 6).

Cardiac fibrosis has been identified as the developing process of heart failure after MI (Wang et al. 2012) and has been involved in p38 MAPK activation (Clerk \& Sugden 2006). Our data demonstrated that all pharmacological interventions reduced p38 MAPK phosphorylation, whereas TGF- $\beta$, a fibrosis inducer, was unchanged. This finding was supported by a previous study which reported that p38 activation could mediate renal fibrosis in a TGF- $\beta$ independent manner (Stambe et al. 2004). Therefore, vildagliptin, metformin, and enalapril given during late-phase $L V$ remodeling in this study could reduce cardiac fibrosis through p38 MAPK signaling in a TGF- $\beta$ independent manner. In addition, NF- $\kappa \mathrm{B}$, a transcription factor that regulates stress and inflammatory response, has been shown to be involved in LV remodeling process (Hamid et al. 2011). It has been shown that an activation of NF- $\mathrm{NB}$ is related with inflammation and postinfarcted LV remodeling after MI (Hamid et al. 2011). In our study, all treatments reduced $\mathrm{NF}-\kappa \mathrm{B}$ activity, suggesting that these drugs reduced inflammation and led to reduce LV remodeling in obese-insulin-resistant rats with chronic MI (Fig. 6).

Growing evidence demonstrates that LV dysfunction is markedly increased in late-phase LV remodeling (Pfeffer et al. 1991, Sutton \& Sharpe 2000). Our results showed that cardiomyocyte hypertrophy and cardiac fibrosis and inflammation are the major contributors of LV dysfunction in our model. Vildagliptin, metformin, and enalapril reduced LV remodeling through several signaling pathways, including reduced ERK1/2, p38 MAPK, and $\mathrm{NF}-\kappa \mathrm{B}$. Moreover, these drugs also reduced oxidative stress and improved cardiac sympathovagal balance. These beneficial effects could be responsible for improved LV function in both ND-fed and obese-insulin-resistant rats with chronic MI (Fig. 6).

It has been shown that an increase of mortality rate after MI is associated with hyperglycemia and cardiac

Published by Bioscientifica Ltd. 


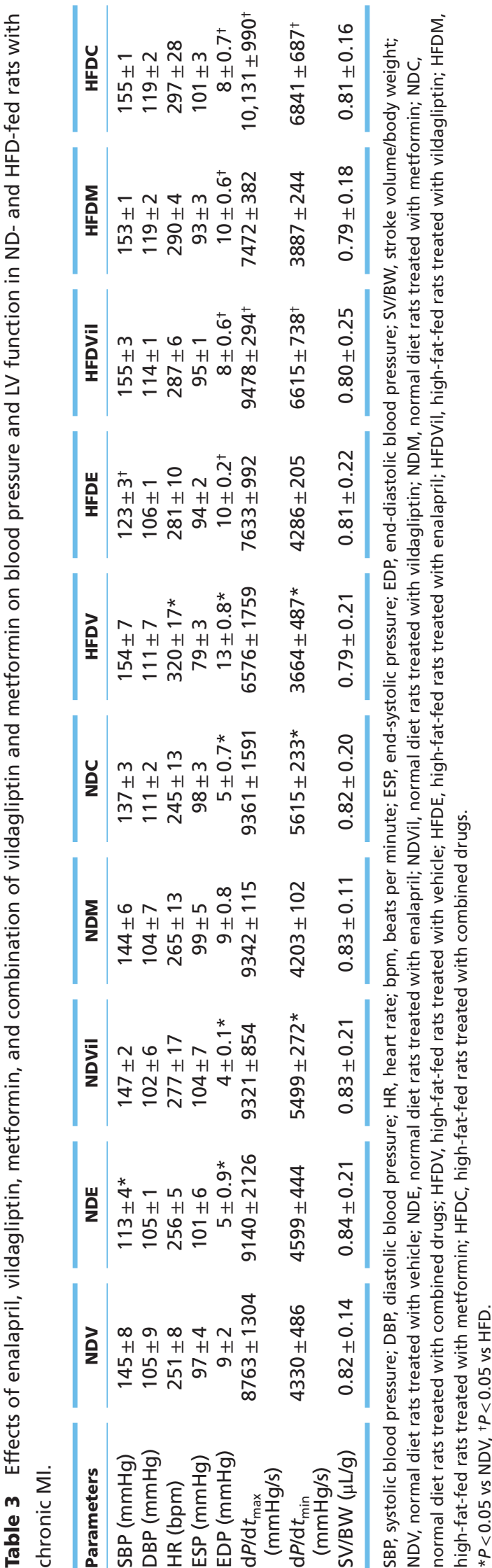

http://joe.endocrinology-journals.org DOI: 10.1530/JOE-16-0096
๑ 2016 Society for Endocrinology Printed in Great Britain sympathovagal imbalance (Chattipakorn et al. 2007, Luo et al. 2014). In this study, the mortality rate was significantly increased in obese-insulin-resistant rats. This finding is also consistent with our previous report in the rat model of ischemia-reperfusion injury (Apaijai et al. 2014). Fatal arrhythmia has been shown to commonly develop and could be responsible for increased mortality in post-MI (Goncalves 1995). CX43 is a gap junction protein that influenced the development of cardiac arrhythmias after MI (Roell et al. 2007). It has been shown that increased CX43 phosphorylation was associated with reduced cardiac arrhythmias (Roell et al. 2007). In this study, all pharmacological interventions reduced hyperglycemia, improved cardiac sympathovagal balance, and increased CX43 phosphorylation, thus leading to decreased mortality in this study (Fig. 6).

In our model, LV remodeling has occurred following MI, leading to LV dysfunction, and the remodeling has developed through these following pathways: (i) fibrosis, (ii) apoptosis, (iii) MAPK pathway that is responsible for cell growth and proliferation, (iv) oxidative stress, and (v) inflammation (Sutton \& Sharpe 2000, Kwak et al. 2006, Hori \& Nishida 2009). In this study, important markers of each pathway were determined to represent the effects of vildagliptin and metformin on LV remodeling such as (i) TGF- $\beta$ as a fibrosis marker, (ii) BAX and BCL2 as apoptosis markers, (iii) p38 and ERK1/2 as markers of cell growth and proliferation, (iv) 8-isoprostane as an oxidative stress marker, and (v) NF- $\mathrm{kB}$ as an inflammatory marker. Enalapril is known to reduce LV remodeling by directly inhibiting the ACE. However, the obese-insulinresistant rats in this study had metabolic disturbances along with LV remodeling after chronic MI. In this study, we demonstrated that although enalapril, vildagliptin, and metformin effectively reduced LV remodeling via those pathways, only vildagliptin and metformin could attenuate the metabolic disturbances in these obeseinsulin-resistant rats. These findings suggested that improvement of metabolic regulation could effectively attenuate adverse LV remodeling in obese-insulinresistant rats with chronic MI.

Apart from the attenuation of insulin resistance, our data also demonstrated that vildagliptin exerted better cardioprotection than metformin through two pathways. First, vildagliptin exerted antioxidant effects in normal rats with chronic MI, whereas metformin could not. Second, vildagliptin reduced cell growth via a reduction of ERK1/2 phosphorylation in normal rats with chronic MI, whereas metformin could not. Therefore, our data suggest that vildagliptin exerted beneficial effects via a reduction

Published by Bioscientifica Ltd. 
A

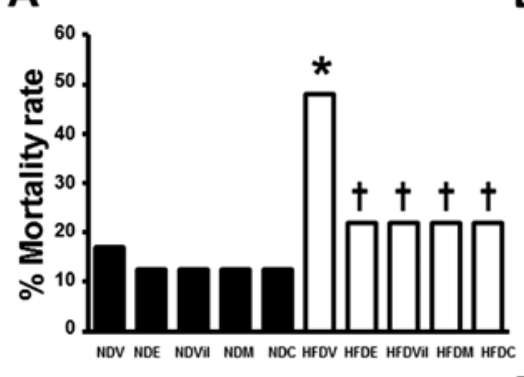

C

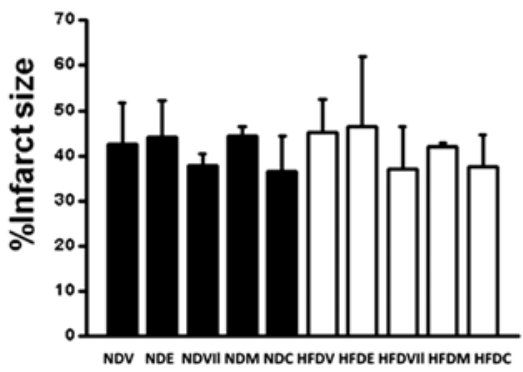

B

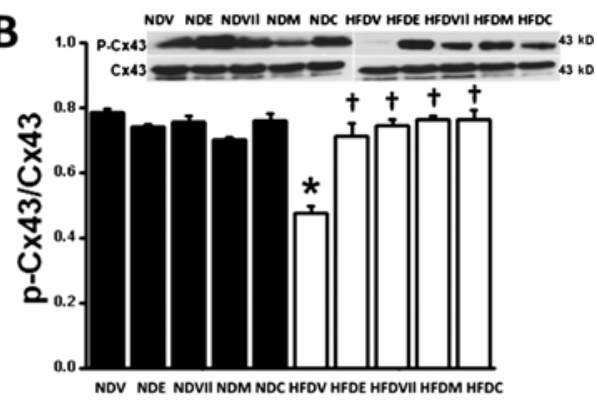

D

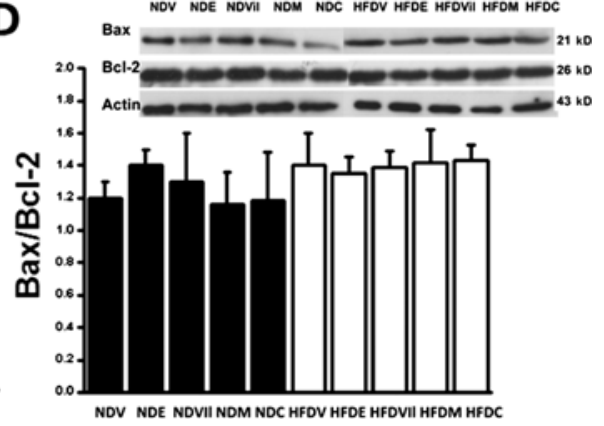

Figure 3

Effects of enalapril, vildagliptin, metformin, and combined drugs on mortality rate, CX43 phosphorylation, infarct size, and BAX/BCL2 ratio in normal and obese-insulin-resistant rats after chronic MI. Mortality rate was higher in HFDV, whereas enalapril, vildagliptin, metformin, and combined vildagliptin and metformin reduced mortality rate $(A)$ and $C X 43$ phosphorylation in obese-insulin-resistant rats after chronic MI (B). ${ }^{*} P<0.05$ vs NDV, ${ }^{+} P<0.05$ vs HFDV. Infarct size (C) and $B A X / B C L 2$ ratio (D) were not different among groups. NDV, normal diet rats treated with vehicle; NDE, normal diet rats treated with enalapril; NDVil, normal diet rats treated with vildagliptin; NDM, normal diet rats treated with metformin; NDC, normal diet rats treated with combined drugs; HFDV, high-fat-fed rats treated with vehicle; HFDE, high-fat-fed rats treated with enalapril; HFDVil, high-fat-fed rats treated with vildagliptin; HFDM, high-fat-fed rats treated with metformin; HFDC, high-fat-fed rats treated with combined drugs.
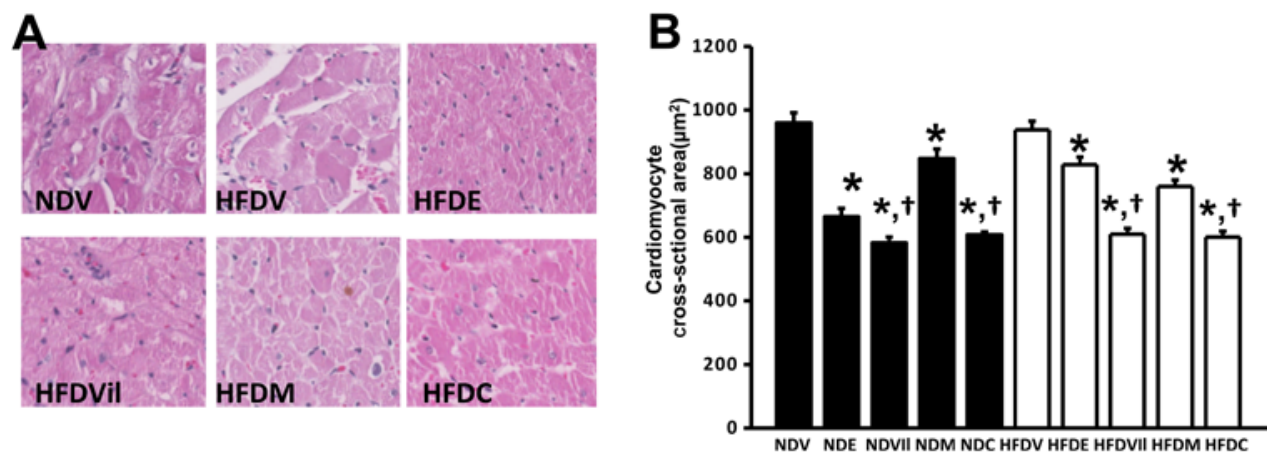

C

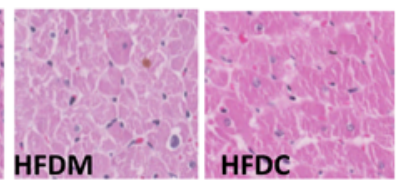

D

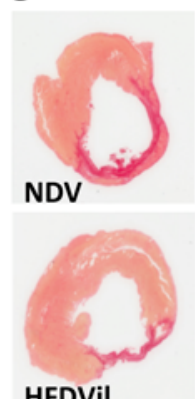

HFDVil

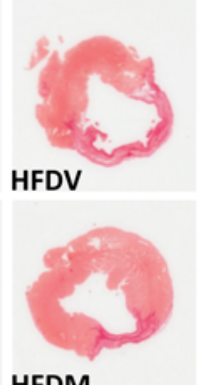

HFDM

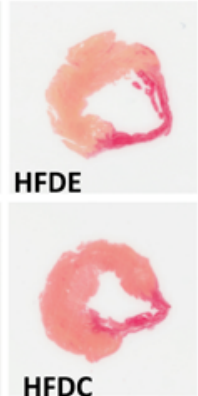

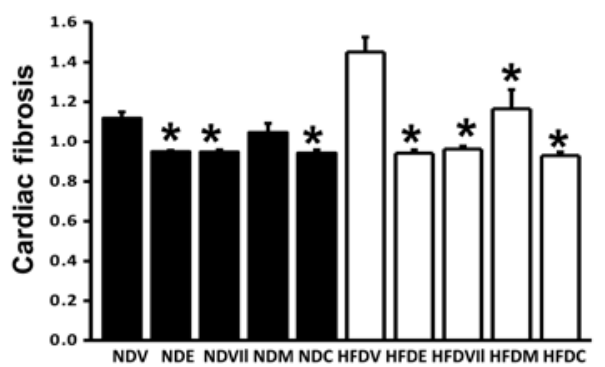

Figure 4

Effects of enalapril, vildagliptin, metformin, and combined drugs on cardiomyocyte cross-sectional area and cardiac fibrosis in normal and obese-insulinresistant rats after chronic MI. Representative picture of cardiomyocyte cross-sectional area from H\&E and picrosirius-stained heart tissue (A and C). Enalapril, vildagliptin, metformin, and combined vildagliptin and metformin decreased cardiomyocyte cross-sectional area in obese-insulin-resistant rats. In ND rats, enalapril, vildagliptin, and combined vildagliptin and metformin but not metformin decreased cardiomyocyte cross-sectional area. However, vildagliptin and combined drugs reduced cardiomyocytes cross-sectional area better than enalapril (B), ${ }^{*} P<0.05$ vs their dietary control, ${ }^{\dagger} P<0.05$ vs enalapril-treated rats within group. Enalapril, vildagliptin, metformin, and combined vildagliptin and metformin reduced cardiac fibrosis in obese-insulin-resistant rats. In ND rats, enalapril, vildagliptin, and combined vildagliptin and metformin but not metformin reduced cardiac fibrosis (D), ${ }^{*}<0.05$ vs their dietary control. NDV, normal diet rats treated with vehicle; NDE, normal diet rats treated with enalapril; NDVil, normal diet rats treated with vildagliptin; NDM, normal diet rats treated with metformin; NDC, normal diet rats treated with combined drugs; HFDV, high-fat-fed rats treated with vehicle; HFDE, high-fat-fed rats treated with enalapril; HFDVil, high-fat-fed rats treated with vildagliptin; HFDM, high-fat-fed rats treated with metformin; HFDC, high-fat-fed rats treated with combined drugs.

http://joe.endocrinology-journals.org
DOI: $10.1530 /$ JOE-16-0096 (c) 2016 Society for Endocrinology Printed in Great Britain
Published by Bioscientifica Ltd 
A nov noe novi nom noc hfov hfoe hfovi hfom hfoc

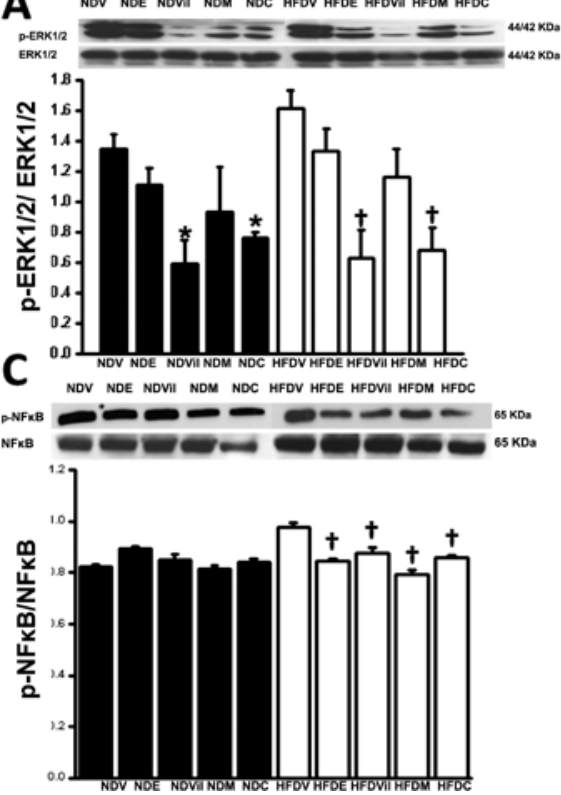

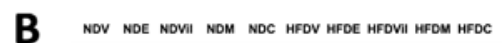

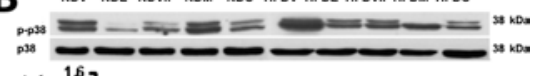

160

D

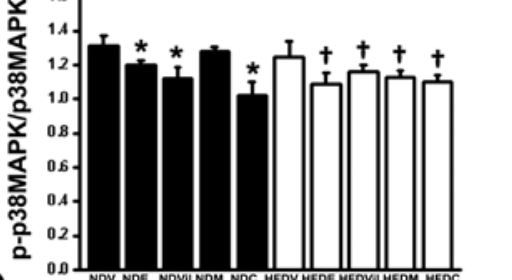

NOV NOE NOVII NOM NOC HFOV HFDE HFOVII HFDM MFDC

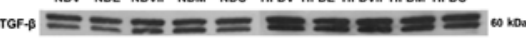
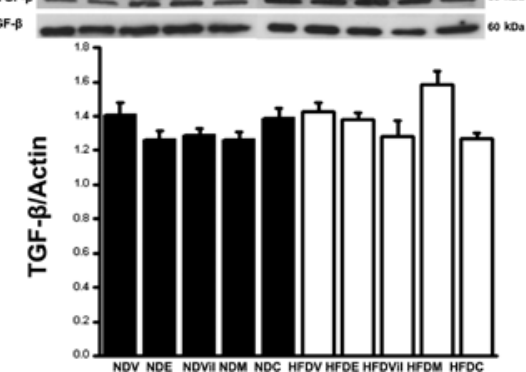

Figure 5

Effects of enalapril, vildagliptin, metformin, and combined drugs on p38MAPK phosphorylation, ERK1/2 phosphorylation, NF- $\mathrm{BB}$ phosphorylation, and TGF- $\beta$ expression in normal and obese-insulinresistant rats after chronic MI. Vildagliptin and combined vildagliptin and metformin reduced ERK phosphorylation in ND and obese-insulinresistant rats (A). Enalapril, vildagliptin, metformin, and combined vildagliptin and metformin reduced p38 MAPK phosphorylation in obese-insulin-resistant rats. In ND rats, enalapril, vildagliptin, and combined vildagliptin and metformin but not metformin decreased reduced p38 MAPK phosphorylation (B). Enalapril, vildagliptin, metformin, and combined vildagliptin and metformin reduced NF-KB phosphorylation in obese-insulin-resistant rats (C). TGF- $\beta$ expression was not different among groups (D), ${ }^{\star} P<0.05$ vs NDV, ${ }^{\dagger} P<0.05$ vs HFDV. NDV, normal diet rats treated with vehicle; NDE, normal diet rats treated with enalapril; NDVil, normal diet rats treated with vildagliptin; NDM, normal diet rats treated with metformin; NDC, normal diet rats treated with combined drugs; HFDV, high-fatfed rats treated with vehicle; HFDE, high-fat-fed rats treated with enalapril; HFDVil, high-fat-fed rats treated with vildagliptin; HFDM, high-fat-fed rats treated with metformin; HFDC, high-fat-fed rats treated with combined drugs. in oxidative stress levels and ERK1/2 phosphorylation in normal rats with chronic MI.

Even though our data indicated that DPP4 inhibitor vildagliptin attenuated adverse LV remodeling in obeseinsulin-resistant rats, the effects of DPP4 inhibitors on HF in the clinical setting are still unclear. At this time, there are five available commercial DPP4 inhibitors: alogliptin, sitagliptin, vildagliptin, saxagliptin, and linagliptin. A report from the EXAMINE trial showed that alogliptin did not increase the risk of a heart failure outcome in T2DM patients (White et al. 2013). For vildagliptin and sitagliptin, the reports from clinical trials still have conflicting data. Some studies reported that sitagliptin and vildagliptin increased neither the risk of adverse

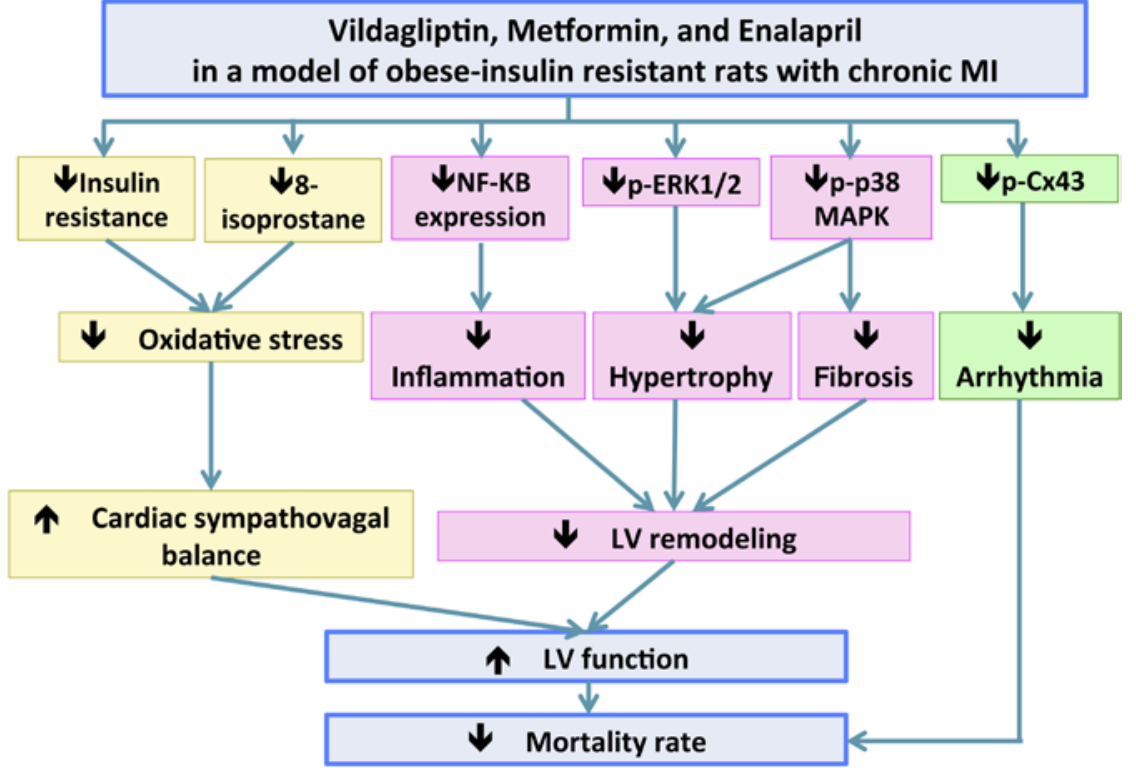

Figure 6

A diagram summarized the effects of vildagliptin, metformin, combined drugs, and enalapil in the obese-insulin-resistant rats with chronic MI. All treatment reduced insulin resistance, reduced oxidative stress, improved cardiac sympathovagal balance, reduced LV remodeling, and increased gap function protein function, leading to improved LV function and reduced mortality rate. CX43, connexin 43 ; LV, left ventricle. A full colour version of this figure is available at http://dx.doi.org/10.1530/ JOE-16-0096. http://joe.endocrinology-journals.org
DOI: 10.1530/JOE-16-0096
๑) 2016 Society for Endocrinology Printed in Great Britain 
cardiovascular events nor the rate of hospitalization for HF (McInnes et al. 2015, Wang et al. 2015, Fu et al. 2016). However, Suh and colleagues reported otherwise, as they showed that sitagliptin and vildagliptin increased the hospitalization rate for $\mathrm{HF}$ after 30 days of medication (Suh et al. 2015). For saxagliptin, the SAVOR-TIMI53 trial demonstrated that saxagliptin increased the rate of hospitalization for heart failure (Scirica et al. 2013). Because these drugs have different forms and different affinities to DPP4, the effect of these DPP4 inhibitors on HF could depend on the type of the drug used in that trial, and this point will need further investigation.

Unlike vildagliptin and enalapril, metformin exerted cardioprotection against MI only in obese-insulin-resistant rats. Previous clinical study reported that metformin did not improve LVEF in ST-elevated MI (STEMI) nondiabetic patients (Lexis et al. 2014a). However, metformin improved clinical outcomes in metabolic syndrome patients undergoing percutaneous coronary intervention (Li et al. 2014) and reduced MI size in diabetic patients with STEMI (Lexis et al. 2014b). Furthermore, metformin has been shown to improve cardiac function in normal rats with chronic MI (Soraya et al. 2012, 2014). All of these findings suggested that metformin exerts cardioprotection in obese-insulin-resistant rats but fails to protect the heart against MI in normal rats. Our findings are consistent with these reports because metformin did not attenuate cardiac autonomic imbalance, LV dysfunction, and LV remodeling in normal rats after MI, whereas it exhibited the beneficial effects in obese-insulin-resistant rats mainly by reducing insulin resistance and exerted cardioprotection after MI in obese-insulin-resistant rats. Data from this study indicated that oxidative stress influenced LV remodeling after MI; metformin did not exert direct antioxidative stress property because metformin did not exert cardioprotection against MI in normal rats. However, vildagliptin exhibited a direct antioxidative stress property in normal rats with MI.

\section{Conclusions}

Vildagliptin, metformin and enalapril attenuated insulin resistance and cardiac sympathovagal imbalance, improved LV function, decreased cardiac fibrosis, and reduced the mortality rate in obese-insulin-resistant rats with MI. However, vildagliptin exerted better efficacy in reducing cardiomyocytes hypertrophy than metformin and enalapril. Moreover, vildagliptin, but not metformin, also provided benefits in improving cardiac sympathovagal balance, reducing cardiomyocyte hypertrophy and cardiac fibrosis, and improving LV function in nonobese-insulinresistant rats with MI.

\section{Declaration of interest}

The authors declare that there is no conflict of interest that could be perceived as prejudicing the impartiality of the research reported.

\section{Funding}

This work was supported by an NSTDA Research Chair Grant from the National Science and Technology Development Agency ( $\mathrm{N} \mathrm{C}$ ), the Thailand Research Fund BRG 5780016 (S C C), the Royal Golden Jubilee program (N C and N A), and a Chiang Mai University Center of Excellence Award (N C).

\section{Acknowledgments}

The authors thank Dr Thanapat Sastraruji for his statistical assistance.

\section{References}

Apaijai N, Pintana H, Chattipakorn SC \& Chattipakorn N 2012 Cardioprotective effects of metformin and vildagliptin in adult rats with insulin resistance induced by a high-fat diet. Endocrinology 153 3878-3885. (doi:10.1210/en.2012-1262)

Apaijai N, Pintana H, Chattipakorn SC \& Chattipakorn N 2013 Effects of vildagliptin versus sitagliptin, on cardiac function, heart rate variability and mitochondrial function in obese insulin-resistant rats. British Journal of Pharmacology 169 1048-1057. (doi:10.1111/ bph.12176)

Apaijai N, Chinda K, Palee S, Chattipakorn S \& Chattipakorn N 2014 Combined vildagliptin and metformin exert better cardioprotection than monotherapy against ischemia-reperfusion injury in obeseinsulin resistant rats. PLOS ONE 9 e102374. (doi:10.1371/journal. pone.0102374)

Bailey CJ 2005 Treating insulin resistance in type 2 diabetes with metformin and thiazolidinediones. Diabetes, Obesity and Metabolism 7 675-691. (doi:10.1111/j.1463-1326.2005.00497.x)

Bui AL, Horwich TB \& Fonarow GC 2011 Epidemiology and risk profile of heart failure. Nature Reviews Cardiology 8 30-41. (doi:10.1038/ nrcardio.2010.165)

Chattipakorn N, Incharoen T, Kanlop N \& Chattipakorn S 2007 Heart rate variability in myocardial infarction and heart failure. International Journal of Cardiology 120 289-296. (doi:10.1016/j.ijcard.2006.11.221)

Chinda K, Palee S, Surinkaew S, Phornphutkul M, Chattipakorn S \& Chattipakorn N 2013 Cardioprotective effect of dipeptidyl peptidase-4 inhibitor during ischemia-reperfusion injury. International Journal of Cardiology 167 451-457. (doi:10.1016/j.ijcard.2012.01.011)

Chinda K, Sanit J, Chattipakorn S \& Chattipakorn N 2014 Dipeptidyl peptidase-4 inhibitor reduces infarct size and preserves cardiac function via mitochondrial protection in ischaemia-reperfusion rat heart. Diabetes and Vascular Disease Research 11 75-83. (doi:10.1177/1479164113516134)

Clerk A \& Sugden PH 2006 Inflame my heart (by p38-MAPK). Circulation Research 99 455-458. (doi:10.1161/01.RES.0000241053.89089.c3)

Derosa G, Bonaventura A, Bianchi L, Romano D, Fogari E, D'Angelo A \& Maffioli P 2014 Vildagliptin compared to glimepiride on post- 
prandial lipemia and on insulin resistance in type 2 diabetic patients. Metabolism 63 957-967. (doi:10.1016/j.metabol.2014.04.008)

Fu AZ, Johnston SS, Ghannam A, Tsai K, Cappell K, Fowler R, Riehle E, Cole AL, Kalsekar I \& Sheehan J 2016 Association between hospitalization for heart failure and dipeptidyl peptidase- 4 inhibitors in patients with type 2 diabetes: an observational study. Diabetes Care [in press]. (doi:10.2337/dc15-0764)

Goncalves L 1995 Risk stratification after myocardial infarction. Clinical evaluation before discharge. Review Port Cardiology 14 475-482, 448.

Gundewar S, Calvert JW, Jha S, Toedt-Pingel I, Ji SY, Nunez D, Ramachandran A, Anaya-Cisneros M, Tian R \& Lefer DJ 2009 Activation of AMP-activated protein kinase by metformin improves left ventricular function and survival in heart failure. Circulation Research 104 403-411. (doi:10.1161/CIRCRESAHA.108.190918)

Hamid T, Guo SZ, Kingery JR, Xiang X, Dawn B \& Prabhu SD 2011 Cardiomyocyte NF-kappaB p65 promotes adverse remodelling, apoptosis, and endoplasmic reticulum stress in heart failure. Cardiovascular Research 89 129-138. (doi:10.1093/cvr/cvq274)

Hori M \& Nishida K 2009 Oxidative stress and left ventricular remodeling after myocardial infarction. Cardiovascular Research 81 457-464. (doi:10.1093/cvr/cvn335)

Inthachai T, Lekawanvijit S, Kumfu S, Apaijai N, Pongkan W, Chattipakorn SC \& Chattipakorn N 2015 Dipeptidyl peptidase-4 inhibitor improves cardiac function by attenuating adverse cardiac remodelling in rats with chronic myocardial infarction. Experimental Physiology 100 667-679. (doi:10.1113/ep085108)

Kiliszek M, Maczewski M, Styczynski G, Duda M, Opolski G \& Beresewicz A 2007 Low-density lipoprotein reduction by simvastatin is accompanied by angiotensin II type 1 receptor downregulation, reduced oxidative stress, and improved endothelial function in patients with stable coronary artery disease. Coronary Artery Disease 18 201-209. (doi:10.1097/ MCA.0b013e32802c7cb0)

Kwak HB, Song W \& Lawler JM 2006 Exercise training attenuates ageinduced elevation in $\mathrm{Bax} / \mathrm{Bcl}-2$ ratio, apoptosis, and remodeling in the rat heart. FASEB Journal 20 791-793.

Lekawanvijit S, Kompa AR, Zhang Y, Wang BH, Kelly DJ \& Krum H 2012 Myocardial infarction impairs renal function, induces renal interstitial fibrosis, and increases renal KIM-1 expression: implications for cardiorenal syndrome. American Journal of Physiology: Heart and Circulatory Physiology 302 H1884-H1893.

Lexis CP, van der Horst IC, Lipsic E, Wieringa WG, de Boer RA, van den Heuvel AF, van der Werf HW, Schurer RA, Pundziute G, Tan ES, et al. $2014 a$ Effect of metformin on left ventricular function after acute myocardial infarction in patients without diabetes: the GIPS-III randomized clinical trial. JAMA 311 1526-1535.

Lexis CP, Wieringa WG, Hiemstra B, van Deursen VM, Lipsic E, van der Harst P, van Veldhuisen DJ \& van der Horst IC 2014b Chronic metformin treatment is associated with reduced myocardial infarct size in diabetic patients with ST-segment elevation myocardial infarction. Cardiovascular Drugs and Therapy 28 163-171.

Li J, Xu JP, Zhao XZ, Sun XJ, Xu ZW \& Song SJ 2014 Protective effect of metformin on myocardial injury in metabolic syndrome patients following percutaneous coronary intervention. Cardiology $\mathbf{1 2 7}$ 133-139.

Liu J, Wang P, Zou L, Qu J, Litovsky S, Umeda P, Zhou L, Chatham J, Marsh SA, Dell'Italia LJ, et al. 2014 High-fat, low-carbohydrate diet promotes arrhythmic death and increases myocardial ischemiareperfusion injury in rats. American Journal of Physiology: Heart and Circulatory Physiology 307 H598-H608.

Luo G, Liu H, Luo S, Li F, Su M \& Lu H 2014 Fasting hyperglycemia increases in-hospital mortality risk in nondiabetic female patients with acute myocardial infarction: a retrospective study. International Journal of Endocrinology 2014745093.
Madian AG, Diaz-Maldonado N, Gao Q \& Regnier FE 2011 Oxidative stress induced carbonylation in human plasma. Journal of Proteomics 74 2395-2416. (doi:10.1016/j.jprot.2011.07.014)

Manzella D, Barbieri M, Ragno E \& Paolisso G 2001 Chronic administration of pharmacologic doses of vitamin $\mathrm{E}$ improves the cardiac autonomic nervous system in patients with type 2 diabetes. American Journal of Clinical Nutrition $\mathbf{7 3}$ 1052-1057.

Mari A, Sallas WM, He YL, Watson C, Ligueros-Saylan M, Dunning BE, Deacon CF, Holst JJ \& Foley JE 2005 Vildagliptin, a dipeptidyl peptidase-IV inhibitor, improves model-assessed beta-cell function in patients with type 2 diabetes. Journal of Clinical Endocrinology and Metabolism $904888-4894$.

McInnes G, Evans M, Del Prato S, Stumvoll M, Schweizer A, Lukashevich V, Shao Q \& Kothny W 2015 Cardiovascular and heart failure safety profile of vildagliptin: a meta-analysis of 17000 patients. Diabetes, Obesity and Metabolism 17 1085-1092. (doi:10.1111/dom.12548)

Miyoshi T, Nakamura K, Yoshida M, Miura D, Oe H, Akagi S, Sugiyama H, Akazawa K, Yonezawa T, Wada J, et al. 2014 Effect of vildagliptin, a dipeptidyl peptidase 4 inhibitor, on cardiac hypertrophy induced by chronic beta-adrenergic stimulation in rats. Cardiovascular Diabetology 1343.

Ocaranza MP, Godoy I, Jalil JE, Varas M, Collantes P, Pinto M, Roman M, Ramirez C, Copaja M, Diaz-Araya G, et al. 2006 Enalapril attenuates downregulation of Angiotensin-converting enzyme 2 in the late phase of ventricular dysfunction in myocardial infarcted rat. Hypertension 48 572-578. (doi:10.1161/ 01.HYP.0000237862.94083.45)

Oh K, Hu FB, Manson JE, Stampfer MJ \& Willett WC 2005 Dietary fat intake and risk of coronary heart disease in women: 20 years of follow-up of the nurses' health study. American Journal of Epidemiology 161 672-679. (doi:10.1093/aje/kwi085)

Pavithran P, Nandeesha H, Sathiyapriya V, Bobby Z \& Madanmohan T 2008 Short-term heart variability and oxidative stress in newly diagnosed essential hypertension. Clinical and Experimental Hypertension 30 486-496. (doi:10.1080/10641960802251875)

Pfeffer JM, Pfeffer MA, Fletcher PJ \& Braunwald E 1991 Progressive ventricular remodeling in rat with myocardial infarction. American Journal of Physiology 260 H1406-H1414.

Pintana H, Apaijai N, Chattipakorn N \& Chattipakorn SC 2013 DPP-4 inhibitors improve cognition and brain mitochondrial function of insulin-resistant rats. Journal of Endocrinology 218 1-11. (doi:10.1530/ JOE-12-0521)

Pipatpiboon N, Pintana H, Pratchayasakul W, Chattipakorn N \& Chattipakorn SC 2013 DPP4-inhibitor improves neuronal insulin receptor function, brain mitochondrial function and cognitive function in rats with insulin resistance induced by high-fat diet consumption. European Journal of Neuroscience 37 839-849. (doi:10.1111/ejn.12088)

Pratchayasakul W, Kerdphoo S, Petsophonsakul P, Pongchaidecha A, Chattipakorn N \& Chattipakorn SC 2011 Effects of high-fat diet on insulin receptor function in rat hippocampus and the level of neuronal corticosterone. Life Sciences 88 619-627. (doi:10.1016/ j.lfs.2011.02.003)

Rachmani R, Lidar M, Brosh D, Levi Z \& Ravid M 2000 Oxidation of low-density lipoprotein in normotensive type 2 diabetic patients Comparative effects of enalapril versus nifedipine: a randomized cross-over over study. Diabetes Research and Clinical Practice $\mathbf{4 8}$ 139-145. (doi:10.1016/S0168-8227(99)00149-7)

Richer C, Fornes P, Cazaubon C, Domergue V, Nisato D \& Giudicelli JF 1999 Effects of long-term angiotensin II AT1 receptor blockade on survival, hemodynamics and cardiac remodeling in chronic heart failure in rats. Cardiovascular Research 41 100-108. (doi:10.1016/ S0008-6363(98)00227-2) http://joe.endocrinology-journals.org

DOI: 10.1530/JOE-16-0096
๑) 2016 Society for Endocrinology Printed in Great Britain 
Roell W, Lewalter T, Sasse P, Tallini YN, Choi BR, Breitbach M, Doran R, Becher UM, Hwang SM, Bostani T, et al. 2007 Engraftment of connexin 43-expressing cells prevents post-infarct arrhythmia. Nature 450 819-824. (doi:10.1038/nature06321)

Scirica BM, Bhatt DL, Braunwald E, Steg PG, Davidson J, Hirshberg B, Ohman P, Frederich R, Wiviott SD, Hoffman EB, et al. 2013 Saxagliptin and cardiovascular outcomes in patients with type 2 diabetes mellitus. New England Journal of Medicine 369 1373-1379. (doi:10.1056/NEJMcibr1308953)

Soraya H, Khorrami A, Garjani A, Maleki-Dizaji N \& Garjani A 2012 Acute treatment with metformin improves cardiac function following isoproterenol induced myocardial infarction in rats. Pharmacological Reports 64 1476-1484. (doi:10.1016/S1734-1140(12)70945-3)

Soraya H, Clanachan AS, Rameshrad M, Maleki-Dizaji N, GhaziKhansari M \& Garjani A 2014 Chronic treatment with metformin suppresses toll-like receptor 4 signaling and attenuates left ventricular dysfunction following myocardial infarction. European Journal of Pharmacology 737 77-84. (doi:10.1016/j.ejphar.2014.05.003)

Stambe C, Atkins RC, Tesch GH, Masaki T, Schreiner GF \& NikolicPaterson DJ 2004 The role of p38alpha mitogen-activated protein kinase activation in renal fibrosis. Journal of the American Society of Nephrology 15 370-379. (doi:10.1097/01. ASN.0000109669.23650.56)

Suh S, Seo GH, Jung CH, Kim MK, Jin SM, Hwang YC, Lee BW \& Kim JH 2015 Increased risk of hospitalization for heart failure with newly prescribed dipeptidyl peptidase- 4 inhibitors and pioglitazone using the korean health insurance claims database. Diabetes Metabolism Journal 39 247-252. (doi:10.4093/dmj.2015.39.3.247)

Sutton MG \& Sharpe N 2000 Left ventricular remodeling after myocardial infarction: pathophysiology and therapy. Circulation 101 2981-2988. (doi:10.1161/01.CIR.101.25.2981)

Takahashi A, Asakura M, Ito S, Min KD, Shindo K, Yan Y, Liao Y, Yamazaki S, Sanada S, Asano Y, et al. 2013 Dipeptidyl-peptidase IV inhibition improves pathophysiology of heart failure and increases survival rate in pressure-overloaded mice. American Journal of Physiology: Heart and Circulatory Physiology 304 H1361-H1369.

Wang J, Huang W, Xu R, Nie Y, Cao X, Meng J, Xu X, Hu S \& Zheng Z 2012 MicroRNA-24 regulates cardiac fibrosis after myocardial infarction. Journal of Cellular and Molecular Medicine 16 2150-2160. (doi:10.1111/j.1582-4934.2012.01523.x)

Wang SH, Chen DY, Lin YS, Mao CT, Tsai ML, Hsieh MJ, Chou CC, Wen MS, Wang CC, Hsieh IC, et al. 2015 Cardiovascular outcomes of sitagliptin in type 2 diabetic patients with acute myocardial infarction, a population-based cohort study in taiwan. PLOS ONE 10 e0131122.

White WB, Cannon CP, Heller SR, Nissen SE, Bergenstal RM, Bakris GL, Perez AT, Fleck PR, Mehta CR, Kupfer S, et al. 2013 Alogliptin after acute coronary syndrome in patients with type 2 diabers. New England Journal of Medicine 369 1327-1335. (doi:10.1056/NEJMoa1305889)

Wong AK, Symon R, AlZadjali MA, Ang DS, Ogston S, Choy A, Petrie JR, Struthers AD \& Lang CC 2012 The effect of metformin on insulin resistance and exercise parameters in patients with heart failure. European Journal of Heart Failure 14 1303-1310. (doi:10.1093/eurjhf/ hfs106)

Yeh CC, Li H, Malhotra D, Turcato S, Nicholas S, Tu R, Zhu BQ, Cha J, Swigart PM, Myagmar BE, et al. 2010 Distinctive ERK and p38 signaling in remote and infarcted myocardium during post-MI remodeling in the mouse. Journal of Cellular Biochemistry 109 1185-1191.

Yin M, Sillje HH, Meissner M, van Gilst WH \& de Boer RA 2011 Early and late effects of the DPP-4 inhibitor vildagliptin in a rat model of postmyocardial infarction heart failure. Cardiovascular Diabetology 1085. (doi:10.1186/1475-2840-10-85)

Zuanetti G, Neilson JM, Latini R, Santoro E, Maggioni AP \& Ewing DJ 1996 Prognostic significance of heart rate variability in postmyocardial infarction patients in the fibrinolytic era. The GISSI-2 results. Gruppo Italiano per lo Studio della Sopravvivenza nell' Infarto Miocardico. Circulation 94 432-436. (doi:10.1161/01.CIR.94.3.432)

Received in final form 29 March 2016

Accepted 30 March 2016

Accepted Preprint published online 4 April 2016
๑) 2016 Society for Endocrinology Printed in Great Britain 